Dorothy Crowfoot Hodgkin: Uma Vida Dedicada à Ciência

Vargas, M. D.*

Rev. Virtual Quim., 2012, 4 (1), 85-100. Data de publicação na Web: 5 de março de 2012 http://www.uff.br/rvq

\title{
Dorothy Crowfoot Hodgkin: A Life Dedicated to Science
}

Abstract: Dorothy Crowfoot Hodgkin was awarded the Nobel Prize in Chemistry 1964 "for her determinations by X-ray techniques of the structures of important biochemical substances". During the $35^{\text {th }}$ Annual Meeting of the Brazilian Chemical Society (SBQ), which took place in Florianópolis from 23 to 26/05/2011, the SBQ paid tribute, in the session Women in Brazilian Chemistry, to four researchers for their contributions to Chemistry in Brazil. Each one in turn paid tribute to the four only women, whose important scientific contributions were recognized with the Nobel Prize in Chemistry since it was created, in 1901. I had the honor and the pleasure of recounting Dorothy Crowfoot Hodgkin's life and work, summarized in this contribution.

Keywords: Dorothy Crowfoot Hodgkin; tribute; X-Ray crystallography.

\section{Resumo}

Dorothy Crowfoot Hodgkin recebeu, em 1964, o prêmio Nobel de Química "por suas determinações, usando técnicas de difração de raios X, das estruturas de importantes substâncias bioquímicas". Na 35a Reunião Anual da Sociedade Brasileira de Química, realizada em Florianópolis de 23 a 26/05/2011, a SBQ homenageou, na sessão Mulheres na Química Brasileira, quatro pesquisadoras por suas contribuições à Química Brasileira. Cada uma, por sua vez, homenageou as únicas quatro mulheres, cujas importantes contribuições científicas foram reconhecidas com a concessão do Prêmio Nobel de Química, desde sua criação, em 1901. Tive a honra e o prazer de apresentar a vida e o trabalho de Dorothy C. Hodgkin, resumidos neste texto.

Palavras-chave: Dorothy Crowfoot Hodgkin; homenagem; cristalografia de raios X.

* Universidade Federal Fluminense, Laboratório de Síntese Organometálica (LSOM), Instituto de Química, Campus do Valonguinho, CEP 24020-150, Centro, Niterói-RJ, Brasil.

M mdvargas@vm.uff.br

DOI: $\underline{10.5935 / 1984-6835.20120007}$

Rev. Virtual Quim. |Vol 4| |No. 1| |85-100| 


\title{
Dorothy Crowfoot Hodgkin: Uma Vida Dedicada à Ciência
}

\author{
Maria D. Vargas *
}

Universidade Federal Fluminense, Laboratório de Síntese Organometálica (LSOM), Instituto de Química, Campus do Valonguinho, CEP 24020-150, Centro, Niterói-RJ, Brasil.

* mdvargas@vm.uff.br

Recebido em 25 de fevereiro de 2012. Aceito para publicação em 4 de março de 2012

\section{Introdução}

\section{Formação}

\subsection{Infância}

2.2. Na Universidade de Oxford

2.3. O doutorado na Universidade de Cambridge

\section{De volta à Universidade de Oxford}

\section{Contribuições seminais}

4.1. Determinação da estrutura da penicilina

4.2. Determinação da estrutura da vitamina B12

4.3. Determinação da estrutura da insulina

\section{Embaixatriz da paz internacional}

\section{Alguns testemunhos}

\section{Introdução}

Na 35a Reunião Anual da Sociedade Brasileira de Química, realizada em Florianópolis de 23 a 26/05/2011, a SBQ homenageou, na sessão Mulheres na Química Brasileira, quatro pesquisadoras, por suas contribuições à Química Brasileira. Cada uma, por sua vez, homenageou as únicas quatro mulheres, cujas importantes contribuições científicas foram reconhecidas com a concessão do Prêmio Nobel de Química, desde sua criação, em 1901 (Figura 1): Marie Curie (em 1911), ${ }^{1}$ Irène Joliot-Curie (em 1935), ${ }^{2}$ Dorothy Crowfoot Hodgkin (em 1964) ${ }^{3}$ e Ada E. Yonath (em 2009). ${ }^{4}$ Coube-me apresentar a vida e o trabalho de Dorothy C. Hodgkin, resumidos no texto que segue; Dorila Piló Veloso (UFMG), Anita Jocelyne Marsaioli (UNICAMP) e Marília Oliveira Fonseca Goulart (UFAL) homenagearam, respectivamente, Marie Curie, Irène Joliot-Curie e Ada E. Yonath.
Dorothy Crowfoot Hodgkin recebeu, em 1964, o prêmio Nobel de Química "por suas determinações estruturais, usando técnicas de difração de raios $X$, de importantes substâncias bioquímicas" incluindo a pepsina, colesterol, lactoglobulina, ferritina, penicilina e a vitamina B-12; mais tarde, desvendou também a estrutura da insulina (à qual se dedicou durante 35 anos). Ajudou a desenvolver e revolucionou uma área importantíssima da ciência, a da cristalografia de proteínas e é conhecida como a fundadora desta ciência fundamental. De fato, o conhecimento da estrutura de proteínas é atualmente de vital importância para o desenvolvimento de novos medicamentos e terapias.

Única mulher a receber o Prêmio Nobel em 1964, em todas as áreas nas quais o Prêmio é concedido, Dorothy Hodgkin manifestou sua esperança de que no futuro próximo, mais mulheres recebessem esta distinção. Desde então, quase meio século se passou, mas somente outras 10 mulheres ganharam o prêmio Nobel de Ciência, entre as quais Ada Yonath. ${ }^{5}$ 

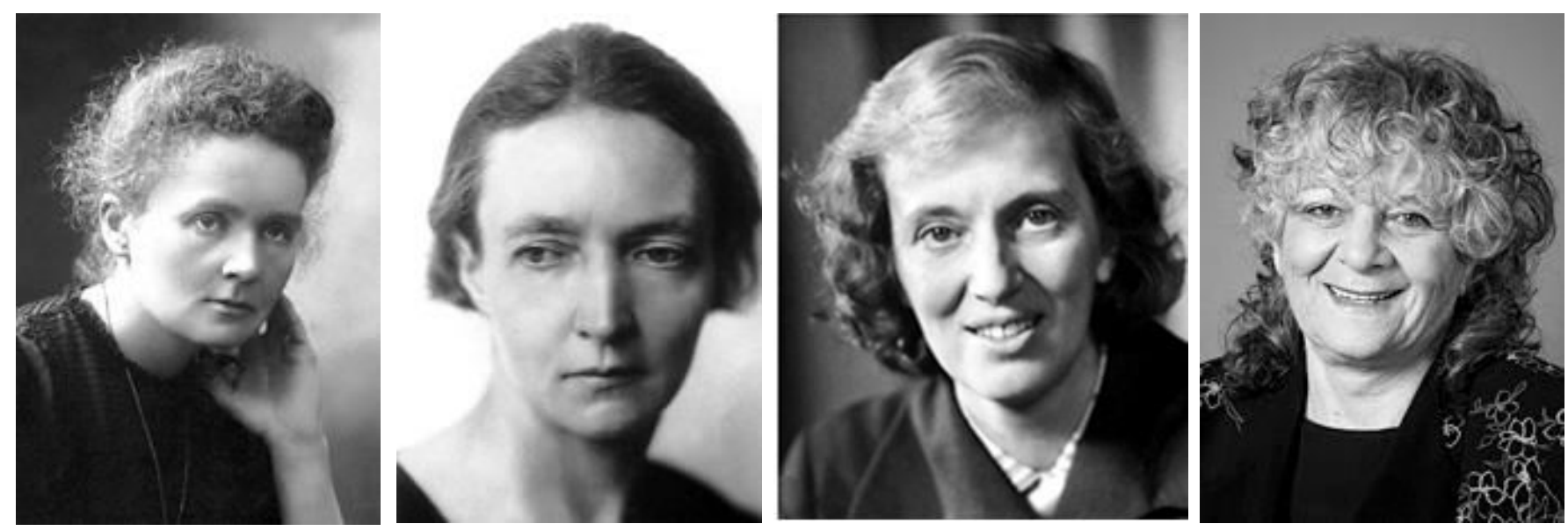

Figura 1. Ganhadoras do Prêmio Nobel de Química: a) Marie Curie (em 1911, sozinha, "em reconhecimento pelas suas descobertas dos elementos rádio e polônio, pelo isolamento do rádio e o estudo da natureza e dos compostos deste elemento notável"; b) Irène Joliot-Curie (em 1935, compartilhou o prêmio com seu marido Jean Frédéric Joliot, "em reconhecimento ao seu trabalho de síntese de novos elementos radioativos"); c) Dorothy Crowfoot Hodgkin (em 1964, sozinha, por "suas determinações, usando técnicas de difração de raios X, das estruturas de importantes substancias bioquímicas") e d) Ada E. Yonath (em 2009, compartilhou o prêmio com Venkatraman Ramakrishnan e Thomas A. Steitz, "por seus estudos sobre a estrutura e o funcionamento do ribossomo")

Dorothy Hodgkin é considerada a mais eminente cientista britânica: única a ter recebido um prêmio Nobel de Química. Foi a primeira mulher, depois de Florence Nightingale (1907), a se tornar Membro da Ordem do Mérito, em 1965 (Figura 2); a primeira mulher a ganhar a Medalha Copley, ${ }^{6}$ a mais antiga e de maior prestígio atribuída pela Royal Society, em 1977, e também a única mulher homenageada pela Royal Society nas comemorações dos seus 350 anos, em 2008, com um selo comemorativo. ${ }^{7}$

Dorothy Hodgkin foi sem dúvida uma mulher excepcional por suas qualidades intelectuais. Em minha apresentação, sobre sua vida e obra, resumida a partir de uma excelente biografia escrita por Georgina Ferry, ${ }^{9}$ extenso material disponível na internet ${ }^{5,10-13}$ e publicado sobre ela ${ }^{14}$ busquei evidenciar como a combinação do seu gênio com as oportunidades criadas pelo seu ambiente familiar e pelas circunstâncias contribuíram para que transcendesse as convenções do seu tempo e alcançasse o reconhecimento máximo.

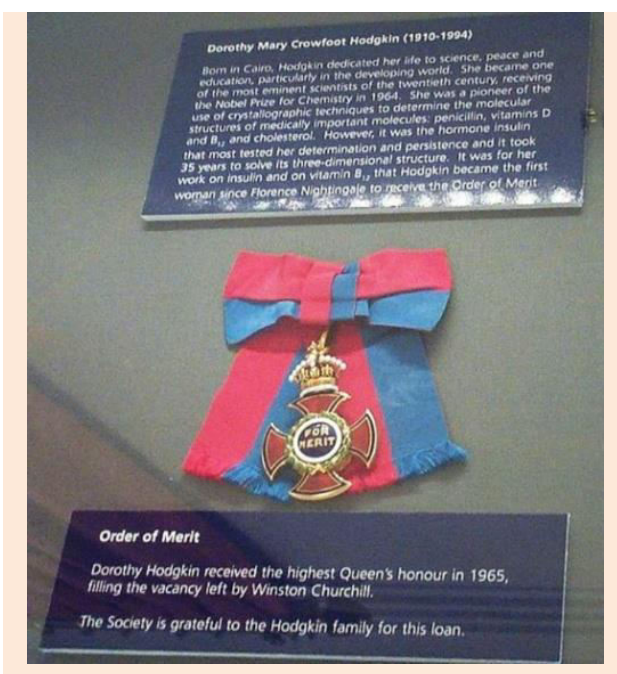

A Ordem do Mérito é uma condecoração britânica e do Commonwealth, outorgada pelo monarca. Foi estabelecida em 1902, como uma recompensa por serviços extraordinários nas Forças Armadas, Ciência, Arte, Literatura ou pela promoção da Cultura.

Dorothy Hodgkin tornou-se Membro da Ordem do Mérito, em 1965, ocupando das vinte e quatro existentes, a vaga de Sir Winston Churchill.

Figura 2. Medalha de Ordem ao Mérito de Dorothy Hodgkin, exibida na Royal Society, em Londres, 20 de Abril de 2004. Fotografia de Kaihsu Tai ${ }^{8}$ 


\section{Formação}

\subsection{Infância}

Dorothy Mary Crowfoot nasceu no dia 12 de maio de 1910 na cidade do Cairo no Egito - um ano antes de Marie Curie ganhar seu segundo prêmio Nobel. Ela foi a primeira das quatro filhas do historiador e educador John Winter Crowfoot, então a serviço da Inglaterra, trabalhando como inspetor de escolas no Egito e depois no Sudão, e Molly Crowfoot. Ambos eram arqueólogos e se não fosse pelo seu fascínio por minerais e cristais, desde a infância, Dorothy certamente teria seguido a vocação dos seus pais.

Quando eclodiu a 1a Guerra Mundial, em 1914, Dorothy e suas irmãs foram mandadas para a casa dos avós, na Inglaterra, onde estariam mais protegidas. Com o fim da guerra, seus pais, vivendo em Cartum, no Sudão, passariam os verões com elas na Inglaterra. De fato, durante os primeiros anos de sua infância Dorothy nunca viveu, por mais que alguns meses, sob o mesmo teto de seus pais. ${ }^{9}$ A esta experiência Dorothy atribuía seu espírito independente.

Aos 11 anos, passou a frequentar uma escola secundária mista, a Leman School, na pequena cidade de Beccles, onde se iniciou na química, através de uma "professora maravilhosa", em suas palavras. ${ }^{9}$ Recorda que foi um experimento de crescimento de cristais de sulfato de cobre que a "cativou pelo resto da vida para a química e os cristais". ${ }^{15}$ Por ocasião de uma visita aos pais em Cartum, aos 13 anos, ganhou de um geólogo amigo da família um laboratório portátil contendo o necessário para realizar análises químicas simples e identificar minerais, que muito usou. Seu dom pela aventura intelectual foi encorajado especialmente pela sua mãe, que vendo seu interesse pelos cristais, permitiu que Dorothy montasse um pequeno laboratório no sótão de casa e Ihe deu livros para expandir seus horizontes. $\mathrm{Na}$ palestra que ministrou ao receber o prêmio Nobel, ${ }^{15}$ Dorothy conta que teve o primeiro contato com a cristalografia de raios $X$ ao ler, aos 16 anos, a versão editada da palestra pública para crianças, ministrada em 1923, por Sir William H. Bragg ${ }^{16}$ da Royal Institution "Sobre a Natureza das Coisas", na qual afirma que a descoberta dos raios $X$ tornou possível "ver átomos e moléculas".

O fato de Molly e John Crowfoot terem tido somente filhas teve influência na educação nada convencional que deram às meninas, e a possível frustração por não terem um herdeiro foi compensada pelas realizações acadêmicas excepcionais de Dorothy. "Fazia parte dos planos do meu pai a meu respeito, que eu fosse educada do mesmo modo que um filho seria e, portanto, que eu estudasse na Universidade de Oxford" (como o pai), lembrava Dorothy. ${ }^{9}$

\subsection{Na Universidade de Oxford}

Dorothy ingressou na Universidade de Oxford em 1928 - ano em que as mulheres conquistaram o direito ao voto - para estudar no Sommerville College, um dos poucos colleges desta Universidade a aceitarem mulheres (Figura 3). Era uma das 5 mulheres entre 60 estudantes de química do seu ano!

Durante os primeiros anos, Dorothy mergulhou em seus estudos de química, à época, essencialmente experimentais, frustrando-se por não the darem a base teórica que explicasse os fatos. Entretanto, soube buscar na biblioteca, através da leitura de trabalhos fundamentais e recentes, por exemplo, de Pauling sobre a natureza da ligação química, e de pesquisadores britânicos que se dedicavam à cristalografia de raios $X$, liderados pelos Bragg, respostas para suas indagações e curiosidade. As aulas de Robert Robinson ${ }^{18}$ a interessavam particularmente, pelo alcance e as conexões que tecia entre a química e a bioquímica. Teve também a oportunidade, durantes os anos de graduação de assistir palestras de vários visitantes ilustres, como Ernest Rutherford, ${ }^{19}$ Niels Bohr, ${ }^{20}$ Peter Debye ${ }^{21}$ e John D. Bernal, ${ }^{22}$ dentre muitos outros, que the causaram forte impressão. ${ }^{9}$ 


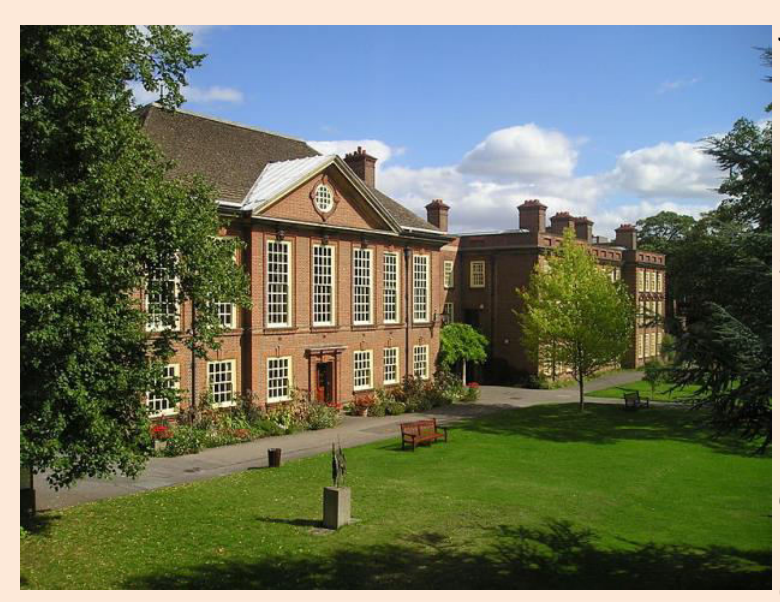

Jovens mulheres tinham poucas oportunidades de estudos na Universidade de Oxford. Embora frequentassem a Universidade nos últimos 50 anos, somente a partir de 1920 puderam ser diplomadas. Mesmo assim, eram comuns professores que não admitiam a presença de mulheres em suas aulas. Um ano antes do ingresso de Dorothy, a Universidade resolveu limitar o número de mulheres para impedir que passasse dos então 840. Por esta razão, Oxford permaneceu pelos 50 anos seguintes uma universidade predominantemente masculina. Sommerville era um dos 5 colleges (dos 27 existentes) dedicados à educação de mulheres. Faziam parte do corpo docente e de tutores destes colleges acadêmicas de alto nível, das áreas mais variadas do conhecimento, que se revezavam para orientar e preparar as alunas para os exames da Universidade.

Figura 3. Sommerville College - Oxford; fotografia de P. Allfrey. ${ }^{17}$ Fundado em 1879 pela "Association for the Higher Education of Women", foi pioneiro na educação superior de mulheres ${ }^{9}$

Após os exames do final do 3ㅇaㅁ ano, estava pronta para desenvolver seu projeto de pesquisa original (chamado de projeto de 4 ㅇ ano, necessário para a obtenção do BA - "Bachelor in Arts"). Por sorte, acabara de ser criado o laboratório de cristalografia de raios $X$, no departamento de Mineralogia. Dorothy optou por esta área (Figura 4), sua primeira paixão, sendo orientada pelo jovem pesquisador H. M. "Tiny" Powell ("Tiny", por que era baixinho), na investigação de estruturas de halogenetos de dialquiltálio(IV). Seu trabalho foi publicado na Nature ${ }^{23}$ e Dorothy graduouse "First Class" em Química, isto é, com a nota máxima. Ela foi a 3 a mulher a ter conseguido este feito na Universidade de Oxford.

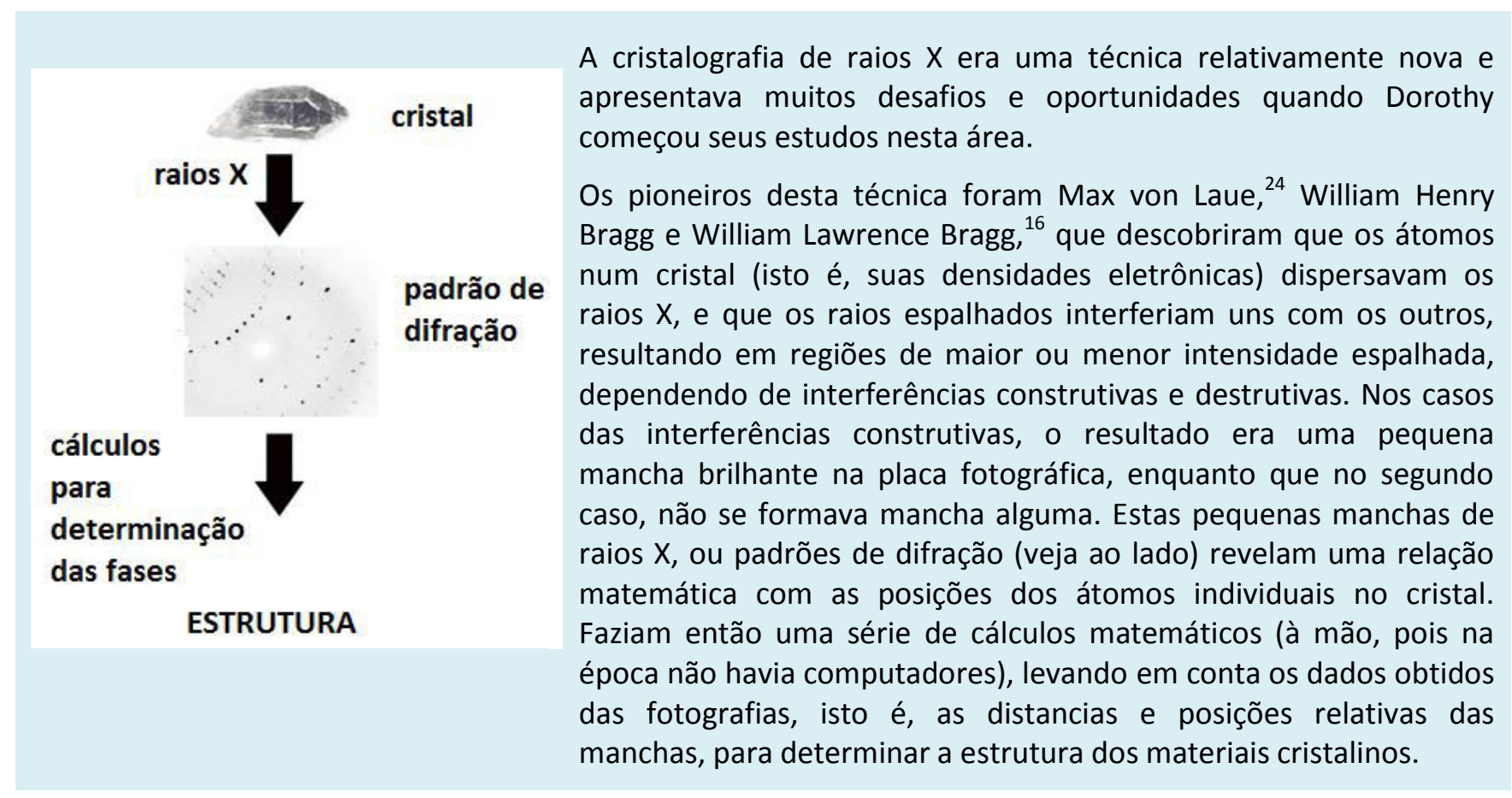

Figura 4. O experimento de difração de raios $X^{25}$ 


\section{2. $O$ doutorado na Universidade de Cambridge}

Na Universidade de Cambridge, onde trabalhou de 1932 a 1934 sob a supervisão de John Desmond Bernal, ${ }^{26-28}$ Dorothy encontrou ambiente estimulante para realizar seu doutoramento. Bernal, cientista de primeira grandeza, havia trabalhado com Bragg pai, e, na época, estava interessado em usar a cristalografia de raios $X$ no estudo estrutural de moléculas biológicas. Apelidado de "Sage" (Sábio), por sua erudição e originalidade, o carismático comunista Bernal apoiava incondicionalmente as mulheres e as encorajava em seu trabalho científico. Dorothy apaixonou-se por ele e por um curto tempo tiveram um affair. ${ }^{9,27}$

Na palestra que ministrou ao receber o prêmio Nobel, Dorothy relata: "Lá (no laboratório de Bernal)... nosso mundo científico não tinha limites... exploramos a cristalografia de uma variedade de produtos naturais, a estrutura de líquidos e particularmente da água, do sal de Rochelle, substituição isomórfica e determinação de fase, cristais de pepsina, e especulamos sobre contração muscular". ${ }^{15}$ A associação com Bernal influenciou fundamentalmente sua carreira, fomentando seu interesse na aplicação da cristalografia de raios $X$ às moléculas biológicas. Além disto, Bernal foi seu mentor científico e amigo, apoiando-a ao longo de sua carreira científica.

A primeira fotografia de uma proteína globular, a pepsina, foi tirada por Bernal. Naquele dia, Dorothy tinha ido consultar um especialista em Londres sobre as dores que vinha sentindo nas mãos, prenúncio da artrite reumatoide que a acompanhou pelo resto da vida. Nos dias seguintes, apesar dos conselhos médicos para que repousasse, Dorothy tirou inúmeras fotografias e realizou as análises que permitiram obter informações sobre sua massa molar e estrutura. Além disto, descobriram que os cristais da proteína tinham de ser estudados embebidos na solução mãe e não secos, como de praxe na época. Esta descoberta, descrita no trabalho que publicaram juntos na Nature, ${ }^{29}$ é considerada o marco inicial da cristalografia de proteínas. Esta técnica, ainda usada nos dias de hoje, permitiu as determinações estruturais da insulina, da hemoglobina, de vírus e do ribossomo, entre outras moléculas.

O tema da sua tese de doutorado foi o estudo cristalográfico dos esteróis, trabalho que continuaria após seu retorno a Oxford. Eram as moléculas mais complexas que já haviam sido estudadas por esta técnica (Figura 5).

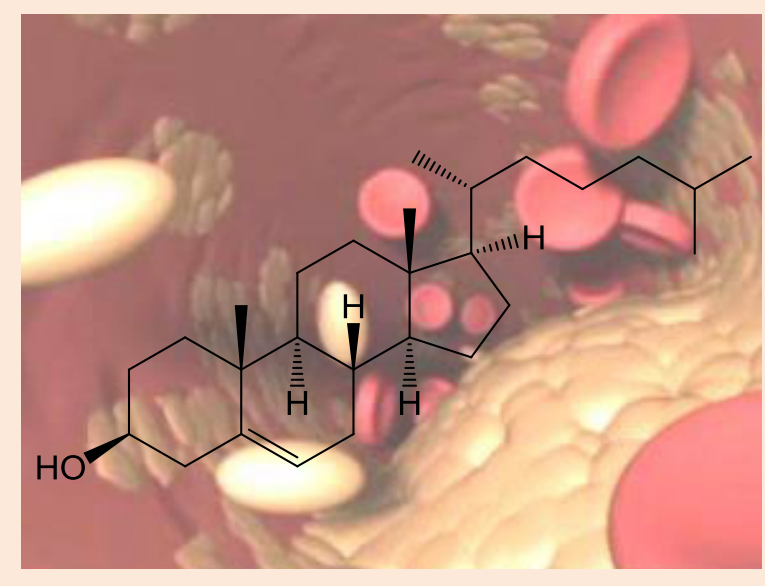

Esteróis são alcoóis policíclicos cuja estrutura básica contém 27 átomos de carbono dispostos em quatro anéis ligados. Exemplos desta classe de moléculas incluem o colesterol (ao lado), o estrogênio e o ergosterol.

Dorothy, com Bernal e Fankuchen estudaram cristais de mais de 100 esteróis, descrevendo as dimensões de suas células unitárias, índices de refração, empacotamento e ligações de hidrogênio em um trabalho monumental, publicado em $1940 .^{5,30,31}$

Figura 5. Estrutura do colesterol

\section{De volta à Universidade de Oxford}

Ao final do seu primeiro ano em Cambridge, Sommerville, seu antigo college de Oxford, Ihe fez uma oferta irrecusável: uma Research Fellowship (bolsa de pesquisa) por dois anos, o primeiro ano, na Universidade de Cambridge, dedicando-se ao seu doutoramento, e no segundo ano, ministrando poucas aulas em Sommerville, para ter tempo de desenvolver sua pesquisa. A associação de Dorothy ao Sommerville college durou por toda sua vida científica: como Fellow e Tutora em Ciências Naturais, envolvida com o ensino da química nos colleges de mulheres, mesmo depois de se tornar Professora (Lecturer) na Universidade de Oxford, em 1946, como Professora Titular (Reader) em cristalografia de raios 
X, em 1956, e finalmente como Wolfson Research Professor da Royal Society, em $1960 .^{9}$

Dorothy transferiu-se para a Universidade de Oxford em 1934. Por ser a única cristalógrafa interessada na determinação estrutural de compostos orgânicos nesta universidade, naturalmente, foi procurada por químicos orgânicos e de produtos naturais, como, por exemplo, o chefe do departamento de Química Orgânica, seu antigo professor, Robert Robinson. ${ }^{18}$ Ele a apoiou, ajudandoa a conseguir um auxílio da ICI (Imperial Chemical Industries) para equipar seu laboratório, situado no porão do Museu de História Natural da Universidade de Oxford, com tubos de raios $\mathrm{X}$ e câmeras de última geração. Deu-lhe também cristais da proteína insulina, que the demandaram três meses de esforços até conseguir obtê-los de tamanho adequado para seus experimentos de difração. Em sua primeira publicação sozinha, na Nature, ${ }^{32}$ Dorothy mostrou que, assim como a pepsina, a insulina poderia ter sua estrutura revelada pela cristalografia. Seu primeiro estudante de pós-graduação, Dennis Riley, em 1937, envolveu-se no trabalho de preparação de cristais de proteínas e investigação dos padrões de difração da lactoglobulina e de várias outras proteínas dentre as quais a insulina que a interessaria durante toda sua vida científica. ${ }^{33}$

Em 1937, no mesmo ano em que defendeu sua tese de doutorado, Dorothy casou-se com Thomas Lionel Hodgkin, como Bernal, um idealista de esquerda e intelectual, na época, envolvido com a educação de adultos. Devido às suas viagens constantes e empregos longe de Oxford, moraram separados até 1945 , quando Thomas foi contratado como professor, em Oxford. Nos anos seguintes, tornou-se grande especialista em estudos africanos, passando longos períodos em universidades estrangeiras, e, em Gana, como consultor do presidente Kwame Nkrumah. ${ }^{34}$ Apesar de não convencional, parece ter sido um casamento feliz. ${ }^{10}$ Tiveram 3 filhos: Luke (1938), Elizabeth (1941) e Toby (1946).

Com o apoio do marido, da família, de amigos e empregados, e do seu college, pode continuar com suas atividades de pesquisa quase ininterruptamente, enquanto vivia a maternidade. Para se ter ideia do ineditismo da situação de Dorothy na Universidade de Oxford, nunca antes uma professora, Fellow em exercício de qualquer college desta Universidade havia tido filho, quando ela engravidou de Luke. Dorothy foi a primeira mulher a gozar de licença maternidade na Universidade de Oxford (paga pelo Sommerville College). Só em 1971 a Universidade passou a conceder este direito às suas professoras! ${ }^{9}$

Rev. Virtual Quim. |Vol 4| |No. 1| |85-100|
Quando teve início a $2^{\mathrm{a}}$ Guerra Mundial, em 1939, Bernal, que havia se transferido no ano anterior para o Birkbeck College, em Londres, passou a colaborar ativamente com o Governo (entre outras contribuições, teve papel fundamental no planejamento da invasão dos aliados na Normandia, em 1944). ${ }^{27}$ Seu laboratório foi evacuado, devido ao perigo de bombardeios e à suas ausências, e quem lucrou com isto foi Dorothy, em Oxford. Bernal transferiu para seu laboratório equipamentos de raios $\mathrm{X}$ mais modernos que acabara de adquirir. Para que Dorothy pudesse continuar suas pesquisas, Bernal solicitou apoio financeiro à Fundação Rockfeller, que a apoiou até os anos 60 ininterruptamente! ${ }^{9}$ Além disto, juntaram-se ao seu time dois competentes colaboradores de Bernal: Dra. Kathe DornbergerSchiff que trabalharia na determinação estrutural de proteínas, e o doutorando Harry Carlisle, envolvido em trabalho sobre esteróis. Com ele, Dorothy determinou, em 1945, a estrutura tridimensional do iodeto de colesterol, ${ }^{35}$ causando grande impacto na comunidade ao demonstrar o potencial da cristalografia para resolver estruturas de moléculas orgânicas complexas.

\section{Contribuições seminais}

Ao longo de sua carreira Dorothy Hodgkin sempre escolheu projetos de grande importância, aqueles que a maior parte dos cientistas da época considerava além do limite do estado da arte. ${ }^{36}$ Tinha capacidade intelectual, insight, intuição feminina e, sobretudo, a persistência para insistir na busca da solução do problema escolhido, mesmo que tivesse de esperar vários anos pela resposta. ${ }^{10}$ Além de contribuir para o desenvolvimento dos métodos usados nas determinações estruturais, determinou, junto com seus alunos e colaboradores, um grande número de estruturas de moléculas importantes do ponto de vista bioquímico e médico.

Escolhi comentar aqui os três estudos considerados referências na área, sobre a determinação estrutural da penicilina, da vitamina B12 e da insulina. Cronologicamente, o primeiro deles envolveu a determinação da estrutura da penicilina, descrita como "o início magnífico de uma nova era da cristalografia". ${ }^{37}$ 


\subsection{Determinação da estrutura da penicilina}

Alexander Fleming descobrira, por acaso, em 1929, que o fungo Penicillium notatum produzia uma substância que impedia seu crescimento, porém avaliara que esta substância era instável demais para ser isolada e usada como fármaco. ${ }^{9}$ Dez anos mais tarde, o patologista Howard Florey e o bioquímico Ernst Chain isolaram a penicilina e comprovaram sua ação antibacteriana. Em 1941, a penicilina já estava sendo usada para tratar soldados feridos na 2 a guerra. Como os métodos de isolamento conhecidos a partir do fungo não permitiam a produção da penicilina em larga escala, era necessário sintetizá-la, mas sem o conhecimento da sua estrutura, era impossível.

Dorothy envolveu-se neste projeto de grande importância militar que estava sendo desenvolvido dos dois lados do Oceano Atlântico por alguns dos químicos orgânicos mais importantes da época. Porém, a estrutura foi desvendada, não através da química e sim da cristalografia, pela jovem Dorothy (Figura 6). Ela obteve cristais do hidrocloreto da penicililamina, em 1942, e junto com sua aluna de doutorado Barbara Low, estudaram os cristais de vários derivados da penicilina. Foi através das análises de difração de raios $X$ dos sais de sódio, potássio e rubídio da benzilpenicilina obtidos nos Estados Unidos, fazendo uso da técnica de substituição isomórfica e de um dos primeiros computadores fabricados no mundo - Hollerith - para fazer os cálculos de Fourier, que chegaram à estrutura tridimensional, em 1945 (Figura 7). Por razões comerciais, este trabalho não pode ser apresentado à comunidade científica neste ano, nas comemorações da Royal Society do cinquentenário da descoberta dos raios $X$ por Röntgen, como Dorothy desejava, e só foi publicado em 1949. ${ }^{38}$

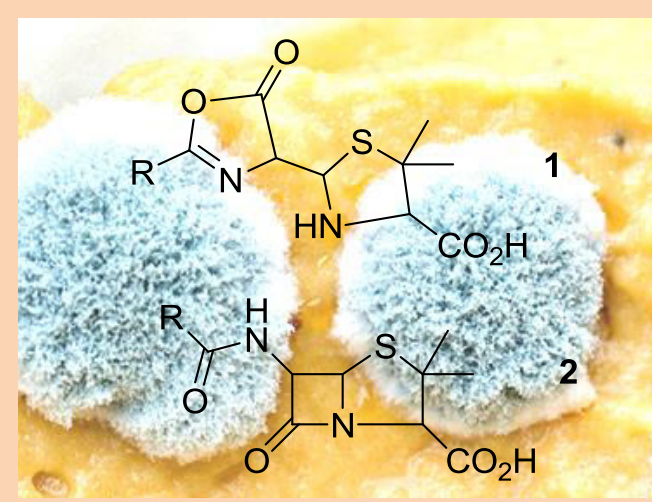

Em 1943 a composição da penicilina ficou conhecida: $\mathrm{C}_{9} \mathrm{H}_{11} \mathrm{~N}_{2} \mathrm{O}_{4} \mathrm{~S}$ Entretanto, sua estrutura tornou-se assunto de debate acalorado. De um lado, Robert Robinson, o Professor de Química Orgânica em Oxford e alguns anos mais tarde Prêmio Nobel de Química, ${ }^{18}$ defendia a estrutura tiazolidina-oxazolona 1, enquanto os bioquímicos Edward Abraham e Ernst Chain favoreciam a estrutura $\beta$-lactama 2. Quando, em 1945, Dorothy e sua aluna de doutorado Barbara Low mostraram que de fato era uma $\beta$-lactama, ${ }^{39}$ Robinson recusou-se a acreditar: não teriam os raios $\mathrm{X}$ alterado a estrutura da penicilina? Foi preciso Dorothy mandar testar a atividade antibacteriana de cristais que haviam sido irradiados com raios $X$ para Ihe mostrar que permaneciam ativos! ${ }^{11}$

Figura 6. Estruturas propostas para a penicilina: tiazolidina-oxazolona $\mathbf{1}$ e $\beta$-lactama $\mathbf{2}$ (a correta, comprovada pelos estudos de difração de raios $\mathrm{X}$ de Dorothy Hodgkin) $;^{36}$ ao fundo o fungo Penicillium notatum 


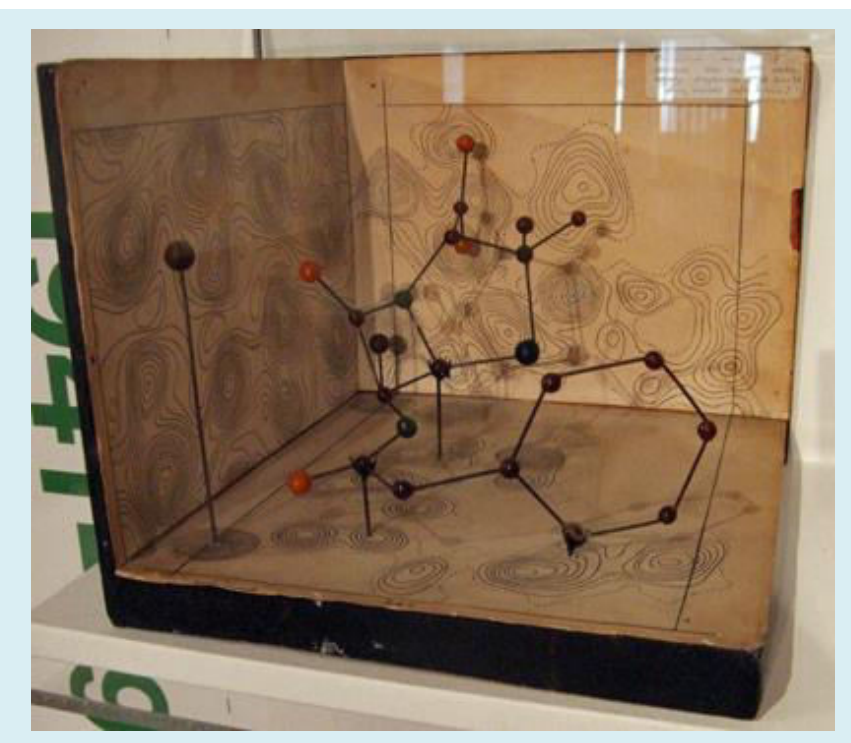

A

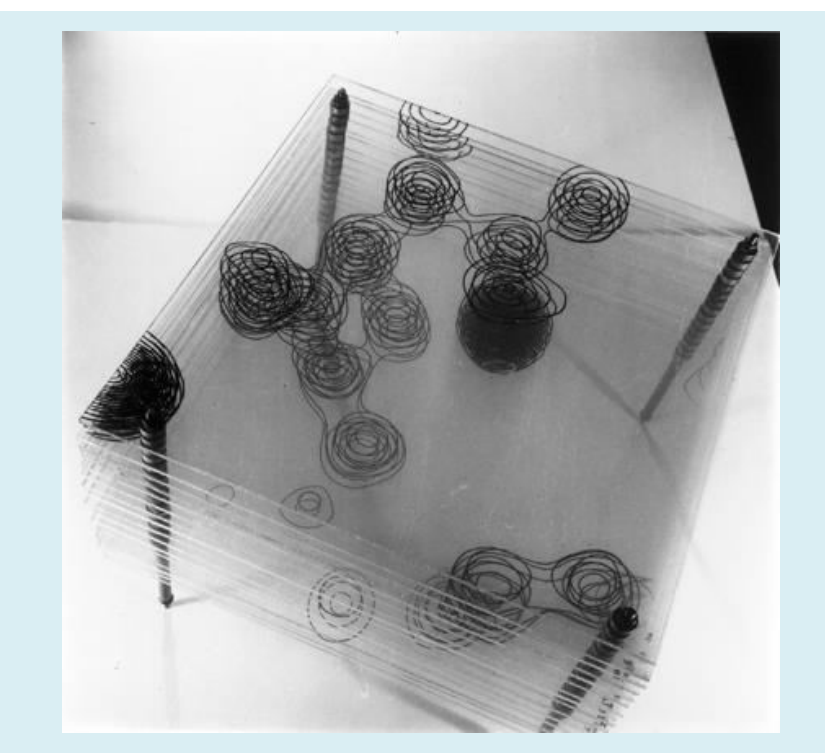

B

Figura 7. Modelos da benzilpenicilina de potássio, feitos por Dorothy C. Hodgkin nos anos 40. A. Modelo fotografado por Matt Brown na Exposição temporária "Penicillin: A story of Triumph and Tragedy at the Science Museum" no Science Museum, Londres, 2007, ${ }^{40}$ e reproduzido com autorização. B Modelo feito em perspex (poli(metacrilato de metila)): os contornos são as linhas de densidade eletrônica e mostram as posições dos átomos individuais na estrutura. ${ }^{41}$ 'Image (C) The Museum of the History of Science, Oxford'

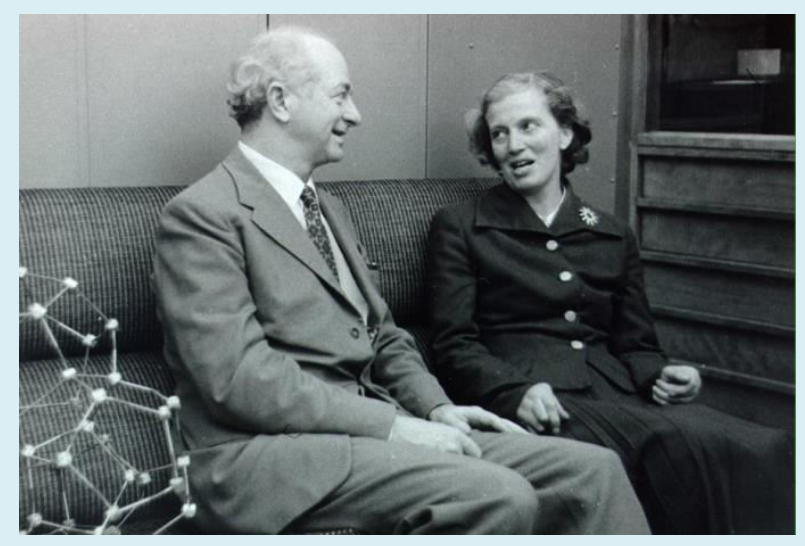

Dorothy conheceu Linus Pauling ${ }^{45}$ por ocasião da visita deste a Oxford, em 1947. A admiração era mútua: Dorothy tinha-o em alta consideração por seu trabalho sobre a ligação química e suas contribuições teóricas à análise estrutural, e Pauling conhecia bem o trabalho de determinação estrutural de Dorothy. Durante sua primeira viagem científica aos Estados Unidos, no mesmo ano, Dorothy passou um mês no CALTEC, com Pauling e colegas. Tornou-se muito amiga dele e da esposa e mantiveram correspondência até o final de suas vidas. ${ }^{46}$ Assim, em 14 de setembro de 1955, Pauling lhe escreveu para "parabenizá-la pelo trabalho maravilhoso sobre a Vitamina B12".

Figura 8. Dorothy Hodgkin com Linus Pauling em 1957. Da Coleção "Ava Helen and Linus Pauling Papers, 18732010", Coleções especiais, Oregon State University ${ }^{46}$

\subsection{Determinação da estrutura da vitamina B12}

A determinação da estrutura da vitamina B12 foi considerada "o triunfo glorioso da análise por cristalografia de raios X", devido tanto à importância química e biológica dos resultados, quanto à imensa complexidade da estrutura dessa molécula. ${ }^{47}$

Sua pesquisa sobre a vitamina B12, então conhecida como fator antianemia perniciosa, teve início em 1948. Sabia-se que a anemia perniciosa, doença fatal, podia ser tratada com extrato de fígado, e foi dele que cristais vermelhos da cianocobalamina (vitamina B12) foram obtidos, na Glaxo, e encaminhados por Lester Smith a Dorothy para estudo. ${ }^{10} \mathrm{Em}$ comparação com as moléculas que já havia investigado, o desafio era imenso: as primeiras fotografias mostraram que a molécula possuía massa molar da ordem de $1500 !^{15}$ Nenhuma das técnicas empregadas anteriormente para analisar os resultados dos mapas de difração era potente o suficiente para enfrentar uma molécula tão grande - 
com mais de cem átomos (sem contar os de hidrogênio). Dorothy e colaboradores tiveram de desenvolver novas técnicas, ainda empregadas atualmente. Além disto, com a colaboração de Ken
Trueblood da Universidade da Califórnia, em Los Angeles (UCLA), usaram computadores para analisar mapas de densidade eletrônica. Após 8 anos de muito trabalho chegaram à estrutura da vitamina B12. ${ }^{48-50}$

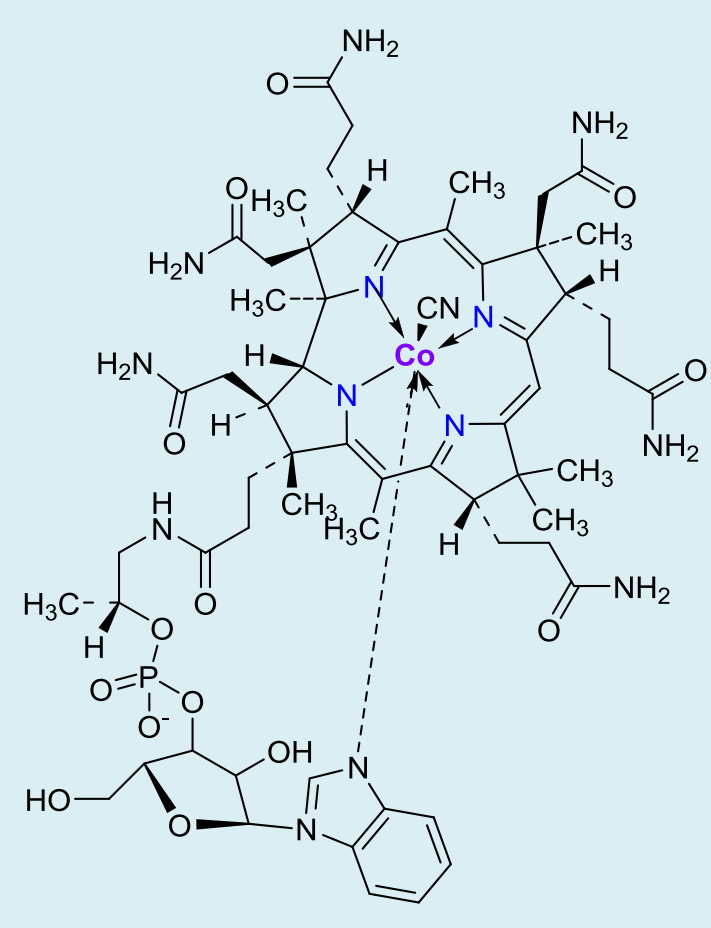

Quando Dorothy iniciou seu trabalho sobre a estrutura da vitamina B12, pouco se sabia sobre sua estrutura química. No final dos anos 40, as investigações de Alexander Todd, em Cambridge, e de outros químicos forneceram informações sobre sua constituição. Demonstraram que a hidrólise ácida desta molécula produzia um grupo do tipo nucleotídeo monofosfato e várias amidas; estudos espectroscópicos mostraram a presença de um grupo cianeto; especulava-se que a molécula continha um anel porfirínico semelhante ao da hemoglobina e da clorofila.

Parte importante da análise da estrutura da vitamina B12 foi a descoberta de que nos cristais, tanto da cianocobalamina, quanto de seus produtos de hidrólise mais simples, o átomo de cobalto se encontrava ligado a um anel corrina, desconhecido até então. Este anel é semelhante ao anel porfirínico, mas contém um átomo de carbono a menos, de modo que dois anéis pirrol ligam-se diretamente. ${ }^{48-50}$

Mais tarde, G. Lenhert e Dorothy mostraram que a coenzima contém uma ligação cobalto-carbono (no lugar da ligação Co-CN na cianocobalamina), o que fez dela o primeiro composto organometálico de ocorrência natural, importante do ponto de vista biológico. ${ }^{51}$

Figura 9. Estrutura da vitamina B12 (cianocobalamina)

William Bragg referiu-se à determinação estrutural desta molécula como "a quebra da barreira do som", querendo dizer com isto que novos horizontes haviam sido abertos no campo da cristalografia. O trabalho levou a várias aplicações médicas, como o tratamento da anemia megaloblástica, e lhe trouxe o prêmio Nobel, 10 anos mais tarde, em $1964 .{ }^{52}$

Este prêmio demorou a chegar. Max Perutz, ${ }^{53}$ seu amigo de muitos anos, conta que "se sentiu envergonhado ao receber o prêmio Nobel antes de Dorothy, cujas grandes descobertas tinham sido realizadas com tão fantástica habilidade e conhecimento de química, e tinham precedido a sua" (descoberta da estrutura da hemoglobina)., ${ }^{9,54}$ Surpreendentemente, apesar da unanimidade entre os pares, a concessão do prêmio Nobel a uma mulher causou surpresa na Inglaterra, como se pode desprender da manchete no jornal britânico, The Daily Mail: "Esposa britânica ganha o prêmio Nobel" ${ }^{55}$ Perutz relata que numa entrevista na radio $B B C$ Ihe perguntaram se ela se sentia em desvantagem na sua carreira por ser mulher. "Para dizer a verdade", respondeu ingenuamente, "os homens sempre foram especialmente gentis comigo por eu ser mulher." ${ }^{56}$

\subsection{Determinação da estrutura da insulina}

A determinação estrutural da insulina foi uma aventura de persistência que se estendeu por toda a vida científica de Dorothy!

A insulina é um hormônio - um mensageiro químico - que controla o metabolismo do açúcar. Foi descoberta em 1921 pelo medico canadense Frederick Banting, pioneiro no uso da insulina no tratamento da diabetes. ${ }^{57}$ Proteínas deste tipo estão entre as moléculas mais complicadas da natureza.

A descoberta por Scott e Fisher, em Toronto, em 1934, de que cristais de insulina continham $\mathrm{Zn}^{2+}$ e que para obter cristais de tamanho adequado era 
necessário adicionar sal deste metal, permitiu a Dorothy obter as primeiras fotografias de difração de cristais da insulina, logo no início de sua carreira independente em Oxford, em 1934. ${ }^{58,59}$ Porém, ninguém imaginava que através da difração de raios $X$ seria possível mapear a estrutura de molécula tão complexa. De fato, na época, as técnicas necessárias para a resolução da sua estrutura ainda não existiam.

Foram necessários 35 anos para que se desenvolvessem os computadores, os equipamentos de difração de raios $X$ e as técnicas de obtenção de cristais que permitiriam obter mapas de densidade eletrônica interpretáveis, a partir dos quais ela e seus colaboradores finalmente derivaram a estrutura, descrita, em 1969, com resolução de 2,8 ̊̊ (Figura 10). A estrutura mostrou o hexâmero (um conjunto de seis moléculas mantidas unidas através de interações fracas) que Dorothy, em seu primeiro artigo de 1934, havia interpretado como sendo "a molécula", na forma de um trímero de dímeros da insulina.

$\mathrm{O}$ artigo foi publicado na Nature, no número que comemorava os 100 anos da revista, assinado por 10 coautores e com agradecimentos a outros 23 pesquisadores que, ao longo dos 35 anos, haviam participado da empreitada. ${ }^{60}$ Entretanto, insatisfeita com o fato de os dados coletados com esta resolução fornecerem a forma da molécula, mas não permitirem definir com exatidão as posições atômicas, Dorothy continuou a trabalhar no problema, mesmo após sua aposentadoria, em 1977. No último trabalho que publicou sobre a insulina, em 1988, aos 78 anos de idade, em coautoria com outros 10 pesquisadores, Dorothy descreveu a estrutura completa com as posições das moléculas de água e resolução de $1,5 \AA{ }^{9}{ }^{9}$, 61

A determinação estrutural deste hormônio foi de tremenda importância. Além de fornecer indícios sobre o seu funcionamento no organismo, permitiu às companhias farmacêuticas produzirem insulina e assim prolongar a vida dos diabéticos. Este trabalho de Dorothy ilustra como ela abordava sua ciência: sua satisfação pessoal dependia da perfeição do seu trabalho. ${ }^{9}$

O interesse de Dorothy pela ciência permaneceu até o final da sua vida, em 1994.

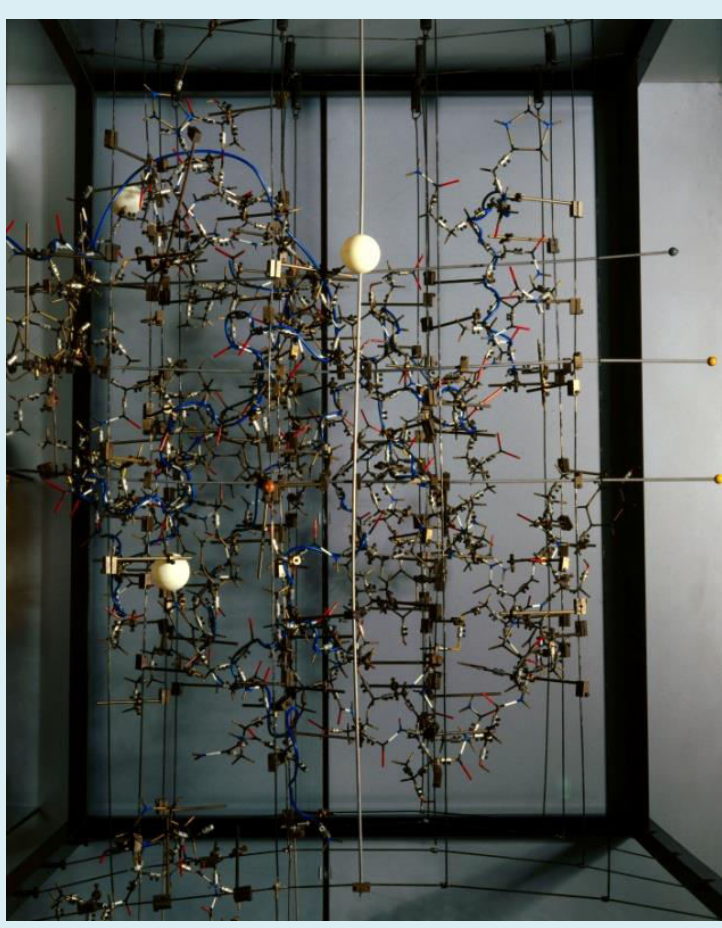

Desde a primeira fotografia da insulina tirada por Dorothy, em 1934, foram 35 anos de trabalho, até chegar à sua estrutura. ${ }^{58,59}$ Seminal foi o trabalho de Fred Sanger ${ }^{62}$ que, em 1955, determinou a sequencia dos 31 grupos de aminoácidos de uma das cadeias e dos 20 da outra. Anteriormente, havia mostrado que as 2 cadeias são mantidas unidas por duas pontes dissulfeto que ele localizara.

Para resolver a estrutura, Dorothy e seu grupo substituíram o $\mathrm{Zn}^{2+}$, utilizado na cristalização da insulina, por $\mathrm{Cd}^{2+}, \mathrm{Pb}^{2+} \mathrm{Hg}^{2+} \mathrm{e}$ uranila $\left(\mathrm{UO}_{2}{ }^{2+}\right)$ e compararam as estruturas alteradas com a original. Conseguiram interpretar as pequenas diferenças entre os cristais e assim neles localizar os átomos pesados. Finalmente, o mapa de densidade eletrônica pode ser interpretado e, baseados na sequencia de aminoácidos de Fred Sanger, construíram o modelo da insulina.

A resolução da estrutura da insulina representou um dos momentos de maior alegria na vida de Dorothy: "Costumava dizer que a noite em que revelei a primeira fotografia da insulina, em 1935, tinha sido o momento mais emocionante da minha vida. Mas o sábado à tarde, no final do mês de julho de 1969, quando me dei conta de que o mapa de densidade eletrônica da insulina poderia ser interpretado chegou muito próximo daquele momento" ${ }^{58}$

Neste modelo da insulina de porco, construído pelo grupo de Dorothy, em 1967, vêm-se as 2 cadeias constituintes da insulina. As esferas maiores representam os átomos de zinco introduzidos quimicamente.

Figura 10. Modelo da insulina de porco que se encontra no Science Museum em Londres. Science Museum/Science \& Society Picture Library, com autorização 


\section{Embaixatriz da paz internacional}

Tendo se tornado figura importante na comunidade científica global, especialmente após receber o prêmio Nobel, Dorothy tentou usar sua influência em prol da paz e da justiça.

Envolveu-se com uma variedade de causas humanitárias, preocupando-se especialmente com o bem estar de cientistas vivendo em países em conflito com os Estados Unidos e a Inglaterra, nos anos 60 e 70, como a União Soviética, a China e o Vietnam. Compartilhava a fé do seu marido e de Bernal no paraíso socialista, e como eles e muitos outros idealistas, atribuía os excessos das ditaduras comunistas aos erros cometidos pelos políticos e não às ideias. De 1976 a 1988 foi presidente da Conferência Pugwash sobre Ciência e Assuntos Mundiais (Pugwash Conference on Science and World Affairs), ${ }^{63}$ originalmente inspirada por Bertrand Russell, ${ }^{64}$ que reúne cientistas influentes e políticos do mundo inteiro preocupados em reduzir o perigo de conflitos armados e em busca de soluções cooperativas para os problemas globais. Dorothy defendeu firmemente os princípios do antimilitarismo, da solução pacífica dos conflitos, dos direitos dos desfavorecidos e da promoção do progresso científico mundial. ${ }^{9} \mathrm{Em} \mathrm{1987,}$, recebeu o prêmio Lenine da Paz, sem dúvida pela sua defesa da causa soviética, mas especialmente pelos seus esforços, como presidente da Pugwash, em aliviar as tensões entre oeste e leste. ${ }^{56}$

Preocupou-se também profundamente com a necessidade de os cientistas reconhecerem os potenciais perigos e as consequências éticas de suas descobertas.

Enquanto ainda estava bastante ativa com sua pesquisa, Dorothy foi também presidente da International Union of Crystallography de 1972 a 1975, e presidente da British Association for the Advancement of Science (o equivalente da nossa SBPC), de 1977 a 1978. Foi também reitora (Chancellor) da Universidade de Bristol de 1970 a 1988.

Um resumo do Currículo de Dorothy, com as honrarias, prêmios, medalhas, doutorados honorários, e academias a que pertenceu pode ser encontrado no sítio eletrônico Contributions of 20th Century Women to Physics, da UCLA. ${ }^{65} 0$ relato completo da vida científica de Dorothy foi publicado na Biographical Memoirs of the Fellows of the Royal Society. ${ }^{11}$

\section{Alguns testemunhos}

A extensa lista de estudantes, colegas e colaboradores de Dorothy inclui os nomes de mais de 110 homens e mulheres de mais de 20 países. Nas palavras de um dos seus colaboradores, Guy Dodson, que ingressou em seu grupo nos anos sessenta, a acompanhou nos esforços da resolução da estrutura da insulina e produziu uma entrevista maravilhosa com Dorothy, lúcida e emocionante, já no final de sua vida:28,31,44 "Em um mundo no qual a ciência frequentemente é feita em grupos grandes e impessoais, Dorothy mostrou que ciência de primeira classe pode ser acompanhada por atenção carinhosa aos valores individuais." ${ }^{\prime 66}$

De acordo com seu amigo Max Perutz: "havia magia em sua pessoa. Não tinha inimigos, nem mesmo aqueles cujas teorias científicas ela havia demolido, ou a cujas opiniões políticas ela se opunha. Era maravilhoso vê-la chegar no laboratório da gente, como a Primavera" ${ }^{67}$ e ainda: "Dorothy foi uma grande química, pura, gentil e tolerante, amante do povo e uma devota protagonista da paz" ${ }^{56}$

\section{Agradecimentos}

A autora agradece à FAPERJ (PRONEX) e ao CNPq (bolsa de pesquisa) e à Sociedade Brasileira de Química pela homenagem.

\section{Referências Bibliográficas}

${ }^{1}$ Sítio do Prêmio Nobel: Química 1911 - Marie Curie. Disponível em: $<$ http://www.nobelprize.org/nobel prizes/chemistry/ laureates/1911/marie-curie.html>. Acesso em: 20 fevereiro 2012.

${ }^{2}$ Sítio do Prêmio Nobel: Química 1935 - Irène JoliotCurie. Disponível em: $<$ http://www.nobelprize.org/nobel prizes/chemistry/ laureates/1935/joliot-curie.html>. Acesso em: 20 fevereiro 2012.

${ }^{3}$ Sítio do Prêmio Nobel: Química 1964 - Dorothy Crowfoot Hodgkin. Disponível em: <http://www.nobelprize.org/nobel prizes/chemistry/ laureates/1964/hodgkin.html>. Acesso em: 20 fevereiro 2012. 
${ }^{4}$ Sítio do Prêmio Nobel: Química 2009 - Ada Yonath. Disponível em: <http://www.nobelprize.org/nobel prizes/chemistry/ laureates/2009/yonath.html>. Acesso em: 20 fevereiro 2012.

${ }^{5}$ Ferry, G. Nature, 2010, 464, 1268. [CrossRef] [PubMed]

${ }^{6}$ Sítio da Royal Society: comemoração dos 350 anos selos. Disponível em: <http://royalsociety.org/RoyalSociety-350th-anniversary-stamps/>. Acesso em: 20 fevereiro 2012.

${ }^{7}$ Sítio da Royal Society: Medalha Copley. Disponível em: <http://royalsociety.org/awards/copley-medal/>. Acesso em: 20 fevereiro 2012.

${ }^{8}$ Sítio da Wilkipédia - Order of Merit Dorothy Hodgkin. Disponível em: <http://pt.wikipedia.org/wiki/Ficheiro:Order of Meri t Dorothy Hodgkin.jpg>. Acesso em: 20 fevereiro 2012.

${ }^{9}$ Ferry, G.; Dorothy Hodgkin: A Life, CSH Press, 2000 ISBN 978-087969594-1.

${ }^{10}$ Glusker, J. P. Prot. Sci. 1994, 3, 2465. [CrossRef] [PubMed]

${ }^{11}$ Dodson, G. G. Biogr. Mems Fell. R. Soc. 2002, 48, 179. [CrossRef]

${ }^{12}$ Sítio The Nobel Prize Internet Archive. Disponível em:

<http://www.almaz.com/nobel/chemistry/1964a.htm I>. Acesso em: 20 fevereiro 2012.

${ }^{13}$ BBC News Audio slideshow: Dorothy Hodgkin. Disponível em: <http://news.bbc.co.uk/2/hi/science/nature/8668708 .stm>. Acesso em: 20 fevereiro 2012.

${ }^{14}$ Gonçalves-Maia, R. Dorothy Crowfoot Hodgkin, Edições Colibri: Lisboa, 2010. ISBN 978-972-991-3.

15 Sítio do Prêmio Nobel: Dorothy Crowfoot Hodgkin Nobel Lecture. Disponível em: <http://www.nobelprize.org/nobel prizes/chemistry/ laureates/1964/hodgkin-lecture.html>. Acesso em: 23 outubro 2011.

${ }^{16}$ Sir William Henry Bragg dividiu o Prêmio Nobel de Física em 1915 com seu filho William Lawrence Bragg por "suas descobertas no ramo da análise de estruturas cristalinas por meio da difração de raios X". Sítio do Prêmio Nobel. Disponível em: <http://www.nobelprize.org/nobel prizes/physics/lau reates/1915/>. Acesso em: 20 fevereiro 2012.

${ }^{17}$ Sítio Wilkipédia sobre Sommerville college.
Disponível

em:

<http://en.wikipedia.org/wiki/Somerville College, Ox ford>. Acesso em: 20 fevereiro 2012.

${ }^{18}$ Robert Robinson ganhou o Prêmio Nobel de Química em 1947 "por suas investigações sobre substancias de plantas de importância biológica, especialmente os alcaloides". Sítio do Prêmio Nobel. Disponível

em:

<http://www.nobelprize.org/nobel prizes/chemistry/ laureates/1947/>. Acesso em: 20 fevereiro 2012.

19 Ernest Rutherford recebeu o prêmio Nobel de Química em 1908 "por suas investigações sobre a desintegração dos elementos e a química de substâncias radioativas". Sítio do Prêmio Nobel. Disponível

em:

<http://www.nobelprize.org/nobel prizes/chemistry/ laureates/1908/rutherford-bio.html>. Acesso em: 20 fevereiro 2012.

${ }^{20}$ Niels Bohr foi agraciado com o Prêmio Nobel de Física, em 1922 por "suas investigações acerca da estrutura dos átomos e da radiação por eles emitida". Sítio do Prêmio Nobel. Disponível em: <http://www.nobelprize.org/nobel prizes/physics/lau reates/1922/bohr-bio.html>. Acesso em: 20 fevereiro 2012.

${ }^{21}$ Peter Debye recebeu o Prêmio Nobel de Química em 1936 por "suas contribuições para o conhecimento da estrutura molecular, através das suas investigações dos momentos de dipolo e da difração de raios X e elétrons em gases". Sítio do Prêmio Nobel. Disponível em: <http://www.nobelprize.org/nobel prizes/chemistry/ laureates/1936/debye.html>. Acesso em: 20 fevereiro 2012.

22 John Desmond Bernal, uma das mentes mais brilhantes do século $X X$, físico, pioneiro na cristalografia de raios $X$. Sítio Public Awareness and Understanding of Science. Disponível em: <http://understandingscience.ucc.ie/pages/sci johnd esmondbernal.htm>. Acesso em: 20 fevereiro 2012.

23 Powell, H. M.; Crowfoot, D. M. Nature, Lond. 1932, 130, 131. [CrossRef]

24 Max Von Laue recebeu o Prêmio Nobel de Física em 1914 por "sua descoberta da difração de raios X por cristais". Sítio do Prêmio Nobel. Disponível em: <http://www.nobelprize.org/nobel prizes/physics/lau reates/1914/>. Acesso em: 20 fevereiro 2012.

${ }^{25}$ Sítio da Wilkipédia sobre cristalografia. Disponível em:

<http://en.wikipedia.org/wiki/X ray crystallography\# Early scientific history of crystals and X-rays>. 
Acesso em: 20 fevereiro 2012.

${ }^{26}$ Hodgkin, D. M. C. Biogr. Mems Fell. R. Soc. 1980, 26, 16. [CrossRef]

${ }^{27}$ Brown, A., J. D. Bernal The Sage of Science, Oxford University Press: Oxford. 2005 - ISBN 978-0-19920565-3

${ }^{28}$ Em entrevista com Guy Dodson, Dorothy expõe as razões que a levaram a trabalhar com Bernal e fala sobre o trabalho em seu laboratório. Sítio da Web of Stories. Disponível no em: <http://www.webofstories.com/play/50707?o=MS>. Acesso em: 20 fevereiro 2012.

${ }^{29}$ Bernal, J. D.; Crowfoot, D. Nature 1934, 133, 794. [CrossRef]

${ }^{30}$ Bernal, J. D.; Crowfoot, D.; Fankuchen, I. Trans. $R$. Soc. 1940, A239, 135. [CrossRef]

${ }^{31} \mathrm{Em}$ entrevista a G. Dodson, Dorothy conta como se desenvolveu o trabalho sobre esteróis. Sítio da Web of Stories. Disponivel em: <http://www.webofstories.com/play/50707?o=MS>, $<$ http://www.webofstories.com/play/50707?o=R> e $<$ http://www.webofstories.com/play/17330?o=S\&srld $=167298>$. Acesso: em 20 fevereiro 2012.

32 Crowfoot, D. Nature 1939, 135, 591. [CrossRef]

${ }^{33}$ Crowfoot, D.; Riley, D. Nature 1939, 144, 1011. [CrossRef]

${ }^{34}$ Sítio da Wilkipédia sobre Thomas L. Hodgkin. Disponível em: $<$ http://en.wikipedia.org/wiki/Thomas Lionel Hodgki n>. Acesso em: 20 fevereiro 2012.

${ }^{35}$ Carlisle, C. H.; Crowfoot, D. Proc. R. Soc. A 1945, 184, 64. [CrossRef]

${ }^{36}$ Dunitz, J. D. Organic chemistry, X-ray analysis, and Dorothy Hodgkin. em: Dodson G., Glusker J. P., Sayre D., eds. Structural studies on molecules of biological interest. A volume in honour of Dorothy Hodgkin. Clarendon Press: Oxford, 1981. pp 47-59.

${ }^{37}$ Hägg, G. Apresentação de Hodgkin, D. C. na cerimônia do Prêmio Nobel. Sítio do Prêmio Nobel. Disponível em:

http://www.nobelprize.org/nobel prizes/chemistry/l aureates/1964/press.html>. Acesso em: 20 fevereiro 2012.

${ }^{38}$ Crowfoot D., Bunn C.W., Rogers-Low B.W., TurnerJones A. X-ray crystallographic investigation of the structure of penicillin. In: Clarke HT, Johnson JR, Robinson R, eds. Chemistry of penicillin. Princeton University Press: Princeton, New Jersey, 1949. pp 310-
367.

${ }^{39}$ Curtis, R.; Jones, J. J. Pept. Sci. 2007, 13, 769. [CrossRef] [PubMed]

${ }^{40}$ Nature.com blog, sobre a Exposição Penicillin at the Science Museum. Disponível em: $<$ http://blogs.nature.com/london/2007/05/11/exhibit ion-penicillin-at-the-science-museum >. Acesso em: 20 fevereiro 2012.

${ }^{41}$ Fox, R. Notes Rec. R. Soc. 2006, 60, 69. [CrossRef]

${ }^{42}$ Equivalente a Membro da Academia de Ciências. Sítio da Royal Society. Disponível em: <http://royalsociety.org/about-us/fellowship/>.

Acesso em: 20 fevereiro 2012.

43 'I remember sitting on the steps of the Royal Society... talking to Bernal and I was telling him that we had solved the structure of penicillin. He said 'you will win the Nobel prize for this', I said 'I would far rather be elected a Fellow of the Royal Society', and he said 'that's more difficult' ref. 9.

${ }^{44}$ Em entrevista com Guy Dodson, Dorothy conta sobre sua viagem aos Estados Unidos, em 1947. Sítio da Web of Stories. Disponível no em: $<$ http://www.webofstories.com/play/17339?o=S\&srld $=167297>$ <http://www.webofstories.com/play/50714?o=MS>. Acesso em: 20 fevereiro 2012.

${ }^{45}$ Linus Pauling recebeu o prêmio Nobel de Química em 1954 e da paz, em 1962. Sítio do Prêmio Nobel. Disponível em: <http://www.nobelprize.org/nobel prizes/chemistry/ laureates/1954/pauling-bio.html> e $<$ http://www.nobelprize.org/nobel prizes/peace/laur eates/1962/>, respectivamente. Acesso em: 20 fevereiro 2012.

${ }^{46}$ The Pauling Blog, mantido pela Universidade de Oregon. Disponível em: $<$ http://paulingblog.wordpress.com/2010/05/11/doro thy-crowfoot-hodgkin-1910-1994/>. Acesso em: 20 fevereiro 2012.

${ }^{47}$ Hägg, G. Apresentação de Dorothy C. Hodgkin na cerimônia do Prêmio Nobel. Sítio do Prêmio Nobel. Disponível em: $<$ http://www.nobelprize.org/nobel prizes/chemistry/ laureates/1964/press.html>. Acesso em: 20 fevereiro 2012.

${ }^{48}$ Brink, C.; Hodgkin, D. C.; Lindsey, J.; Pickworth, J.; Robertson, J. H.; White, J. G. Nature 1954, 174, 1169. [CrossRef] [PubMed] 'The determination of the structure of vitamin $B 12$, has been considered the crowning triumph of X-ray crystallographic analysis, 
both in respect of the chemical and biological importance of the results and the vast complexity of the structure'

${ }^{49}$ Hodgkin, D. C.; Pickworth, J.; Robertson, J. H.; Trueblood, K. N.; Prosen, R. J. White, J. G. Nature 1955, 176, 325. [CrossRef] [PubMed]

${ }^{50}$ Dorothy conta, em entrevista a Guy Dodson, a saga da determinação da estrutura da vitamina B12. Sítio da Web of Stories. Disponível em: $<$ http://www.webofstories.com/play/17340?o=S\&srld $=167298>$,

$<$ http://www.webofstories.com/play/17342?o=S\&srld $=167298>$,

$<$ http://www.webofstories.com/play/17343?o=S\&srld $=167298>$

$\langle$ http://www.webofstories.com/play/17344?o=MS>. Acesso em: 20 fevereiro 2012.

${ }^{51}$ Lenhert, P. C.; Hodgkin, D. C. Nature 1961, 192, 937. [CrossRef] [PubMed]

52 Vídeo da entrega do Prêmio Nobel a Dorothy C. Hodgkin, em 1964. Sítio do Prêmio Nobel. Disponível em:

$<$ http://www.nobelprize.org/mediaplayer/index.php? id=1033 > . Acesso em: 20 fevereiro 2012.

${ }^{53}$ Max Ferdinand Perutz recebeu o prêmio Nobel de Química em 1962, junto com John Cowdery Kendrew "por seus estudos sobre as estruturas de proteínas globulares". Sítio do Prêmio Nobel. Disponível em: <http://www.nobelprize.org/nobel prizes/chemistry/ laureates/1962/\#>. Acesso em: 20 fevereiro 2012.

${ }^{54}$ Perutz, M. Forty years friendship with Dorothy em Structural Studies of Molecules of Biological Interest, Dodson, G.; Glusker, J.P.; Sayre, D. eds. Oxford University Press: Oxford. 1981. "I felt embarrassed when I was awarded the Nobel Prize before Dorothy, whose great discoveries had been made with such fantastic skill and chemical insight, and had preceded my own."

${ }^{55}$ The Daily Mail's headline was "British wife wins Nobel Prize". Ref 9

${ }^{56}$ Perutz, M. Dorothy Crowfoot Hodgkin - Obituary. Sítio da International Union of Crystallography. Disponível em: $<$ http://www.iucr.org/people/crystallographers/dorot hy-crowfoot-hodgkin-by-m.f.-perutz>. Acesso em: 20 fevereiro 2012.

${ }^{57}$ Frederick Grant Banting recebeu o prêmio Nobel de Fisiologia ou Medicina junto com John James Rickard Macleod "pela descoberta da insulina". Sítio do Prêmio Nobel. Disponível em: <http://www.nobelprize.org/nobel prizes/medicine/l aureates/1923/banting-bio.html\#>. Acesso em: 20 fevereiro 2012.

${ }^{58}$ Hodgkin, D. C. Br. Med. J. 1971, 4, 449. [CrossRef] [PubMed] "I used to say the evening that I developed the first $x$-ray photograph I took of insulin in 1935 was the most exciting moment of my life. But the Saturday afternoon in late July 1969, when we realized that the insulin electron density map was interpretable, runs that moment very close."

${ }^{59} \mathrm{Em}$ entrevista com Guy Dodson, Dorothy conta sobre seu trabalho com a insulina Sítio da Web of Stories Disponivel em: $<$ http://www.webofstories.com/play/17329?o=MS > e $\langle$ http://www.webofstories.com/play/17348?o=MS $>$. Acesso em: 20 fevereiro 2012.

${ }^{60}$ Adams, M. J.; Blundell, T. L.; Dodson, E. J.; Dodson, G. G.; Vijayan, M.; Baker, E. N.; Harding, M. M.; Hodgkin, D.; Rimmer B.; Sheat, S. Nature 1969, 224, 491. [CrossRef]

${ }^{61}$ Baker, E. N.; Blundell, T. L; Cutfield, J. F.; Cutfield, S. M.; Dodson, E. J.; Dodson, G. G.; Hodgkin, D. M. C.; Hubbard, R. E.; Isaacs, N. W.; Reynolds, C. D.; Sakabe, K.; Sakabe, N.; Vijayan, N. M. Phil. Trans. R. Soc. Lond. $B$ 1988, 319, 369. [CrossRef]

${ }^{62}$ Frederick Sanger recebeu o prêmio Nobel de Química em 1958 "por seu trabalho sobre a estrutura de proteínas, especialmente a da insulina". Sítio do Prêmio Nobel. Disponível em: <http://www.nobelprize.org/nobel prizes/chemistry/ laureates/1958/>. Acesso em: 20 fevereiro 2012.

${ }^{63}$ Sítio da Pugwash Conferences on Science and World Affairs. Disponivel em: $<$ http://www.pugwash.org/>. Acesso em; 20 fevereiro 2012.

${ }^{64}$ Bertrand Russell recebeu o prêmio Nobel de Literatura em 1950 "em reconhecimento por seus variados e significantes escritos nos quais defende ideais humanitários e a liberdade de pensamento." Disponível em: $<$ http://www.nobelprize.org/nobel prizes/literature/l aureates/1950/>. Acesso em: 20 fevereiro 2012.

65 Sítio "Contributions of 20th Century Women to Physics" - UCLA - Dorothy Crowfoot Hodgkin. Disponível em: $<$ http://cwp.library.ucla.edu/Phase2/Hodgkin, Dorot hy Crowfoot@841234567.html>. Acesso em: 20 fevereiro 2012.

${ }^{66}$ Dodson G., Glusker J. P., Sayre D., eds. Structural studies on molecules of biological interest. A volume in 
honour of Dorothy Hodgkin. Clarendon Press: Oxford, 1981. "In a world in which science is often performed in large impersonal groups, Dorothy has shown that absolutely first-class science may still be joined with a loving attention to individual values."

${ }^{67}$ Sutton, M. Chemistry World, 2010, July, 62-65.
"There was magic about her person. She had no enemies, not even among those whose scientific theories she demolished or whose political views she opposed. It was marvelous to have her drop in on you in your lab, like the spring." 